\title{
Sacral Neuromodulation in Patients With a Cardiac Pacemaker
}

\author{
Abdullah A. Gahzi, Dean S. Elterman, Magdy Hassouna \\ Department of Urology, Toronto Western Hospital, Toronto, Canada
}

\begin{abstract}
The objective of this study was to describe our experience using sacral neuromodulation to treat urinary urgency, frequency, urge incontinence, and chronic urinary retention in patients with cardiac pacemakers. With the increasingly widespread use of InterStim for bladder function restoration, we are seeing more complex patients with multiple comorbidities, including cardiac conditions. Herein, we report 3 cases of individuals with cardiac pacemakers who underwent InterStim implantation to treat urinary conditions. This study is a case series of 3 patients with cardiac pacemakers who underwent sacral neuromodulation to treat refractory voiding dysfunction. The initial patient screening for InterStim therapy involved percutaneous nerve evaluation (PNE), in which a temporary untined lead wire was placed through the S3 foramen. Patients who did not respond to PNE proceeded to a staged implant. All patients in this study had a greater than 50\% improvement of their urinary symptoms during the initial trial and underwent placement of the InterStim implantable pulse generator (IPG). Postoperative programming was done under electrocardiogram monitoring by a cardiologist. No interference was observed between the InterStim IPG and the cardiac pacemaker. In this group of patients, sacral neuromodulation in the presence of a cardiac pacemaker appears to have been safe.
\end{abstract}

Keywords: Cardiac Pacemakers; Implantable Pulse Generator; Sacral Neuromodulation; Urge Urinary Incontinence; Urinary Retention

- Research Ethics: The study design was provided to the research ethics board and they decided that formal approval was not necessary. The application for institutional authorization submitted was reviewed, and the study design was determined not to require institutional authorization under UHN Policy 40.20 .008$.

- Conflict of Interest: No potential conflict of interest relevant to this article was reported.

The incidence of urinary urgency, frequency, and urge incontinence increases with age, with an estimated prevalence of $16.9 \%$ in women and $16 \%$ in men [1]. Conservative therapy is aimed at behavioral modifications, including timed voiding, bladder retraining, and double voiding. Pharmacologic therapy with either an antimuscarinic or beta- 3 agonist may be used as second-line therapy. The use and efficacy of these therapeutic modalities may be limited in patients with refractory symptoms or with other medical conditions, such as limited mobility, glaucoma, or cardiac disease, which may make adherence to such therapy difficult or contraindicated [2].

Sacral neuromodulation via InterStim therapy (Medtronic Inc., Minneapolis, MN, USA) has been approved by the Food and Drug Administration and Health Canada for the treatment of refractory overactive bladder, frequency-urgency syndrome, and chronic urinary retention. It involves the implantation of a quadripolar lead into the third sacral foramen adjacent to the third sacral nerve. The electrode is attached to an implantable pulse generator (IPG), which is implanted in the subcutaneous tissue of the buttock. The mechanism of action of sacral neuro-
Corresponding author: Magdy Hassouna (D) http://orcid.org/0000-0002-1096-3391 Department of Urology, Toronto Western Hospital, 399 Bathurst St, Toronto, ON, Canada

E-mail: Magdy.Hassouna@uhn.ca / Tel: +1-416-603-5018 / Fax +1-4166031961 Submitted: February 17, 2016 / Accepted after revision: April 4, 2016
This is an Open Access article distributed under the terms of the Creative Commons Attribution Non-Commercial License (http://creativecommons.org/licenses/by-nc/4.0/) which permits unrestricted non-commercial use, distribution, and reproduction in any medium, provided the original work is properly cited. 
modulation is not well understood, but it primarily modulates the pelvic floor and bladder function by inducing electric pulses to the sacral nerves, principally through the sensory component of the sacral reflex arc. To determine if a patient is a candidate for implantation, a stimulation test trial is performed. Patients who show a response to the test $(\geq 50 \%$ improvement in one or more of the voiding symptoms from baseline) are considered candidates for implantation. The test trial can either involve a percutaneous nerve evaluation (PNE), which is an outpatient test, or a staged procedure performed in the operating room.

Patients are increasingly living longer lives with chronic conditions as a result of advances in medical technology. Cardiac pacemakers have become commonplace, while sacral neuromodulation continues to grow as a therapeutic option for refractory voiding dysfunction. Some theoretical concerns remain that the electrical stimulation created by the InterStim IPG may interfere with the electrical signal of a cardiac pacemaker. Herein, we report a series of 3 patients who underwent InterStim implantation for refractory voiding symptoms with cardiac pacemakers in situ.

\section{CASE REPORT}

\section{Methods}

The patients included in this study were seen for refractory voiding dysfunction at the Division of Urology at Toronto Western Hospital (Toronto, ON, Canada). The study design was provided to the research ethics board and they decided that formal approval was not necessary. The application for institutional authorization submitted was reviewed, and the study design was determined not to require institutional authorization under UHN Policy 40.20.008.

All patients underwent a complete history and physical examination, including the relevant laboratory investigations. Urodynamic studies are not routinely performed before InterStim implantation; however, 1 patient in this series did undergo urodynamic studies as part of the initial evaluation.

The patients were given a 3- to 5-day voiding diary prior to the PNE. Patients who showed a $\geq 50 \%$ improvement in one or more of the voiding symptoms from baseline were considered candidates for implantation.

Patients who did not respond to the PNE proceeded to a staged implant in the operating theater under general anesthesia. Lead placement was confirmed by motor response in con- junction with anteroposterior and lateral views on fluoroscopy.

In order to carefully monitor patients with preexisting cardiac pacemakers, postoperative programming was performed under electrocardiogram (ECG) and pacemaker programmer monitoring by a cardiologist or a pacemaker clinic clinician. The testing process included increasing the pacemaker sensitivity and the output amplitude of the InterStim neuromodulator. The sacral neuromodulation IPG was programmed to a strictly bipolar output when cardiac pacemakers utilized bipolar sensing. No instances were observed of the cardiac pacemaker turning off spontaneously or of disruptions or alterations to the sensing response.

\section{Patients}

Patient 1 was a 66-year-old female known to have bradycardia secondary to lithium cardiomyopathy with a cardiac pacemaker. She presented with urgency, urge incontinence, nocturia 1-2 times per night, and enuresis. At the time of her examination, she was wearing 30 pads per day. Behavioral, antimuscarinic, and beta-3 agonist therapy had not succeeded. Her medical history included glaucoma, gastroesophageal reflux, hypercholesterolemia, hypothyroidism, bronchial asthma, colitis, and bipolar disorder. She responded to the PNE and the InterStim was implanted. This apparatus controlled her daytime symptoms, but she still reported nocturia and enuresis. Desmopressin was prescribed, but her symptoms still did not improve. Over the last 2 months of InterStim therapy, the stimulation became painful and she decided to have the InterStim removed. Ultimately, she had the InterStim in place for 10 months.

Patient 2 was a 67-year-old male with a history of hypertension, ischemic heart disease, and bradycardia with a cardiac pacemaker. He presented with a frequency of every 1-2 hours, urgency, and nocturia 3-5 times per night. Behavioral, alphablocker, 5-alpha-reductase inhibitor, antimuscarinic and beta-3 agonist therapy had previously been attempted and found to be unsuccessful. A urodynamic study confirmed the presence of uninhibited detrusor contractions. He responded to PNE, and the InterStim was implanted. Postoperative programming using bipolar settings was performed under ECG monitoring. No effects on his cardiac rhythm or pacemaker sensing were noted. The patient remained satisfied with the implant at a 6-month follow-up.

Patient 3 was a 67-year-old female with bradycardia treated with a cardiac pacemaker. She presented with the inability to completely empty her bladder, with occasional urgency, urge 
incontinence, and nocturia 1-2 times per night. She could urinate on her own, although she self-catheterized 3-4 times per day and drained approximately $200 \mathrm{~mL}$ each time. She experienced occasional episodes of fecal incontinence. Previous behavioral and alpha-blocker therapy had not been successful. Her medical history included bronchial asthma, gastroesophageal reflux, hypercholesterolemia, hypertension, depression, and deep vein thrombosis. She had previously undergone a sigmoidectomy for benign disease. The PNE failed and she proceeded to a staged implant. Postoperative programming using bipolar settings was performed under ECG monitoring. No effects were observed on her cardiac rhythm or on pacemaker sensing. The patient remained satisfied with the implant at a 6-month follow-up.

\section{DISCUSSION}

Refractory urinary urgency, frequency, and urge incontinence are indications for sacral neuromodulation with InterStim therapy [3]. Case reports presented by Iyer et al. [4] and Andersen et al. [5] have stated that the simultaneous use of spinal cord stimulators and cardiac pacemakers was safe. Wallace et al. [2] reported 3 patients with cardiac pacemakers who underwent staged implants. The quadripolar lead wire was implanted under local anesthesia with continuous cardiac monitoring under anesthesia and in the presence of a cardiac pacemaker technician. Maximal stimulation of the electrode was then done to identify any interference with cardiac pacing. The patients were then observed in a telemetry unit for 24 hours. The patients were monitored during the second stage and the first round of programming postoperatively. Additionally, Roth [6] reported 5 patients with cardiac pacemakers who underwent staged implants under local sedation. Intraoperative cardiac monitoring and postoperative telemetry did not demonstrate cross-interference with the test stimulation.

This report describes the outcomes of 3 patients with cardiac pacemakers who underwent sacral neuromodulation and implantation of the InterStim pulse generator for refractory urgency, frequency, urge incontinence, and chronic urinary retention. Patients underwent continuous cardiac monitoring during pacemaker programming by a cardiologist or pacemaker clinic clinician. The settings used for programming were pulse widths of $210 \mu \mathrm{sec}$, a rate of $14 \mathrm{~Hz}$, and an amplitude of up to $10 \mathrm{~V}$. No instances of inhibition or interference with cardiac pacing were observed, even when maximal sacral nerve stimulation was applied to each of the 4 electrodes along the lead wire.

Additionally, all 3 patients had bipolar cardiac pacemakers, which may have minimized potential interference from electrical or electromagnetic stimulation [7].

We conclude that InterStim sacral neuromodulation appears to be safe in patients with cardiac pacemakers programmed using bipolar settings. Continuous cardiac monitoring and pacemaker telemetry are recommended during programming, as well as testing using maximal sacral nerve stimulation, in order to ensure that no inhibition or interference occurs.

\section{ACKNOWLEDGEMENTS}

We would like to thank Mr. James Steeves, a Medtronic representative who carried out the programming for our patients in this study.

\section{REFERENCES}

1. Stewart WF, Van Rooyen JB, Cundiff GW, Abrams P, Herzog AR, Corey R, et al. Prevalence and burden of overactive bladder in the United States. World J Urol 2003;20:327-36.

2. Wallace PA, Lane FL, Noblett KL. Sacral nerve neuromodulation in patients with cardiac pacemakers. Am J Obstet Gynecol 2007; 197:94.e1-3.

3. Siegel SW, Catanzaro F, Dijkema HE, Elhilali MM, Fowler CJ, Gajewski JB, et al. Long-term results of a multicenter study on sacral nerve stimulation for treatment of urinary urge incontinence, urgency-frequency, and retention. Urology 2000;56(6 Suppl 1):87-91.

4. Iyer R, Gnanadurai TV, Forsey P. Management of cardiac pacemaker in a patient with spinal cord stimulator implant. Pain 1998; 74:333-5.

5. Andersen C, Oxhøj H, Arnsbo P. Management of spinal cord stimulators in patients with cardiac pacemakers. Pacing Clin Electrophysiol 1990;13:574-7.

6. Roth TM. Sacral neuromodulation and cardiac pacemakers. Int Urogynecol J 2010;21:1035-7.

7. Irnich W. Interference in pacemakers. Pacing Clin Electrophysiol 1984;7(6 Pt 1):1021-48. 